\title{
CRONOLOGÍA POR TL DEL YACIMIENTO NEOLÍTICO DE "EL ESPINO" (BARRANCO DEL DURATÓN, SEGOVIA)
}

\section{A TERMOLUMINISCENCE DATE OF “EL ESPINO" NEOLITHIC SITE (BARRANCO DE DURATÓN, SEGOVIA)}

\author{
por \\ $M^{a}$ ROSARIO LUCAS \\ LUZ Ma CARDITO \\ ELLEN ETZEL \\ RAFAEL ANCIONES \\ ILDEFONSO RAMÍREZ
}

\begin{abstract}
RESUMEN La datación de cinco muestras de cerámicas, efectuada por el sistema de Termoluminiscencia (TL), permite precisar la cronología y el contexto cultural del yacimiento de "El Espino" (Barranco del Duratón, Segovia).
\end{abstract}

\begin{abstract}
The dating of five samples of ceramics using the thermoluminiscent system gives the exact chronology and cultural context of the neolithic site of "El Espino" (Barranco del Duratón, Segovia).
\end{abstract}

Palabras claves El Espino, Neolítico antiguo, Duratón, Segovia, Cronología

Key words $\quad$ El Espino, Early Neolithic, Duratón, Segovia, Chronology

El abrigo de "El Espino" está emplazado en el Parque Natural de las Hoces del Duratón, en el paraje denominado Cuesta de la Hoz (término municipal de Villaseca, Segovia) Se trata de una oquedad natural abierta en el estrato medio de la pared del barranco que bordea la margen derecha del.río Duratón, frente a las ruinas del Monasterio de Nuestra Señora de la Hoz. Como referencias más explícitas cabe señalar que se localiza en cota más baja y a mitad de camino, entre la Cueva de la Nogaleda y el Solapo del Águila (fig. 1)

En artículos anteriores se ha hecho ya referencia a la existencia y descubrimiento fortuito de este abrigo y en esta ocasión queremos dar a conocer la novedad de las fechas aportadas por $\mathrm{TL}^{\mathrm{L}}$, contribuyendo al

1. Lucas et alii (1997); Lucas et alii (2001: publicación en CD): lamentablemente las notas se han suprimido en esta edición. En una de ellas se adelantaba la cronología del Abrigo del Espino. 
merecido homenaje dedicado al Dr. Pellicer cuyos trabajos en las cuevas granadinas marcaron en su momento una inflexión en el estudio del Neolítico Español, campo en el que nuestro homenajeado es referencia obligada por su continuada labor y contribución al Neolítico en Andalucía.

Del yacimiento de El Espino sólo tenemos conocimiento visual del sitio: un abrigo como tantos otros del barranco ( $18 \mathrm{~m}$ de boca $\mathrm{x} 3 \mathrm{~m}$ de alto y unos $2 \mathrm{~m}$ de profundidad). La terraza natural actúa de piso o suelo, protegido en su momento por la visera desprendida, caída a plomo, documentada en la actualidad por las enormes piedras que cubren casi por completo el suelo de la oquedad.

En una de las prospecciones del barranco, con el objetivo de localizar arte rupestre ${ }^{2}$, reparamos en la existencia de unos pocos fragmentos cerámicos que se extendían superficialmente alrededor y en los márgenes de las pesadas piedras; una somera capa de tierra, aparentemente débil, de coloración y textura diferente a la cubierta vegetal, es el único indicio que nos permite suponer la existencia de un nivel arqueológico.

Al pie de las rocas caídas se localizaron también tres piezas líticas y, rodados por la ladera, otros restos cerámicos, alcanzando el cómputo total de la muestra cerámica veintidós fragmentos. Sus características decorativas confirman que se trata de cerámica neolítica en su mayoría decorada con fuertes impresiones (entre ellas la gradina: fig. 2:7) que todavía conservan, pese a la erosión, incrustación de pigmento rojo muy degradado (fig. 2:8,11 y 12 y fig. 3: 14 y 20) e incluso blanco (fig. 2:6), acanaladuras e incisiones, sin faltar fragmentos lisos e incluso la decoración plástica de mamelones (fig. 2:4 y 3:18), con posibles acabados "a la almagra", enmascarados o muy perdidos por el desgaste de las superficies En conjunto remiten a recipientes de tamaño medio, con predominio de cuencos, y sólo, lo que puede ser una base convexa gruesa (no dibujada) podría pertenecer a una vasija de almacenaje.

En términos generales, las características de estos materiales no difieren de otros hallazgos segovianos dentro del barranco, especialmente de la serie cerámica procedente de la Cueva de la Nogaleda o Nogalera (Municio y Ruiz Gálvez 1986) o de la no lejana Cueva de la Vaquera en Torreiglesias (Estremera 1999) y, lo que es también importante, de otros conjuntos cerámicos del interior de la Meseta fuera de Segovia (Jiménez Guijarro 1998 y 1999; Kunst y Rojo 1999) En este sentido, y aunque carezcamos de datos sobre la naturaleza del yacimiento, más allá de su inmediatez al arte rupestre (Lucas et alii 1997; 2001), la datación absoluta por Termoluminiscencia (TL) puede ser de gran ayuda para ordenar en el tiempo la presencia y expansión de los grupos humanos productores de alimentos cuyas manifestaciones materiales se asocian recurrentemente a estas modalidades cerámicas.

\section{LAS MUESTRAS}

Las cinco fechas disponibles, a partir de otros tantos fragmentos, se han obtenido en 1999 en el Laboratorio de Datación y Radioquímica de la Universidad Autónoma de Madrid. De acuerdo a sus indicaciones (especialmente de la Dra. Millán) se seleccionó para el muestreo la arcilla del núcleo, descartando la capa/película exterior, con el fin de que la pasta que iba a determinar el periodo de cocción de la cerámica, estuviera lo más exenta posible de contaminaciones externas. Asimismo se proporcionó al laboratorio arcilla de la margen del río y algún fragmento calizo de las rocas del entorno.

El protocolo/analítica, según Informe del Laboratorio, que transcribimos literalmente, ha sido el siguiente:

— Selección de muestras evitando, en la medida de lo posible, su exposición a la luz y otras fuentes de radiación.

2. Proyecto dirigido por $\mathrm{M}^{\mathrm{a}} \mathrm{S}$. Corchón y Financiado por la Comunidad de Castilla y León y el Ministerio de Cultura. 
- Hecha la selección, aislamiento con el fin de evitar procesos de evaporación, así como de exposición a la luz.

— Finalmente, se llevaron a cabo las medidas radiométricas.

Las referencias del laboratorio a las cinco muestras analizadas corresponden a:

\begin{tabular}{|l|c|}
\hline \multicolumn{1}{|c|}{$\begin{array}{c}\text { REF. YACIMIENTO } \\
\text { El Espino (Barranco del Duratón) }\end{array}$} & $\begin{array}{c}\text { REF. LABORATORIO } \\
\text { Dat. /Radioquímica U.A.M. }\end{array}$ \\
\hline Mad- 1.Cerámica Mamelón (Inv. 18) & MAD - 1048 \\
\hline Mad- 2. Cerámica Lisa (Inv. 19) & MAD - 1049 \\
\hline Mad- 3. Cerámica Incisa (Inv. 20) & MAD - 1050 \\
\hline Mad- 4.Cerámica con Colorante (Inv. 11) & MAD - 1051 \\
\hline Mad- 5. Cerámica Impresa (Inv. 6) & MAD - 1052 \\
\hline
\end{tabular}

\section{MANIPULACIÓN DEMUESTRAS Y PROCEDIMIENTO PARA LA REALIZACIÓN DE MEDIDAS}

Todas las muestras seleccionadas fueron sometidas a un test previo de decaimiento anómalo, dicho estudio fue realizado a partir de la respuesta de Tl (sistema Riso Tl-DA-10) obtenida de las muestras en un segundo barrido, después de ser almacenadas en oscuridad durante un período de tiempo de 240 horas. De tal modo, que cuando las pérdidas de señal detectadas son inferiores al $3 \%$, dicho test se considera negativo, o, en otras palabras, el posible fenómeno de decaimiento anómalo se considera insignificante.

Sobre la base de estos resultados, el método de datación por TL seleccionado fue el de grano-fino (Zimmerman 1971), consistente en una selección de la fracción mineral con tamaño de grano comprendido

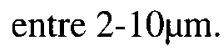

La dosis total almacenada por cada muestra desde que sufrió su último calentamiento (dosis arqueológica) fue evaluada a través del método de dosis aditivas; dichas dosis crecientes fueron suministradas mediante una fuente beta de $\mathrm{Sr}-\mathrm{Y}^{90}$ con una tasa de dosis de $0.1488 \mathrm{mGy} / \mathrm{sg}$. Con el objeto de determinar un posible comportamiento supralineal se realizó un segundo barrido, con dosis beta pequeñas (Fleming 1975) La efectividad de las partículas alfa para producir TL (valor-K) fue determinada mediante el suministro de dosis alfa crecientes, mediante la utilización de una fuente de $\mathrm{Am}^{241}$, con una tasa de dosis de $0,0297 \mathrm{mGy} / \mathrm{sg}$. Todas las respuestas de TL fueron obtenidas después de un calentamiento previo de las muestras, a $90^{\circ}$ C durante $120 \mathrm{seg}$, con el fin de eliminar las señales inestables de TL. Los cálculos de la dosis arqueológica y el valor-K fueron obtenidos en la región de temperaturas correspondiente al "plateau" de la curva resultante de la representación de TL natural/TL inducida frente a la temperatura (Aitken 1985)

El cálculo de las dosis-anuales recibidas por las muestras fue realizado mediante la combinación de dos tipos de medidas, por un lado la determinación de la radioactividad beta procedente del K- 40 presente en las muestras, mediante un sistema de recuento Geiger-Müller, y por otra la medida de la actividad alfa procedente del Uranio y Torio, también presentes en las muestras, en este caso usando un sistema de recuento de centelleo sólido (ZnS) En este último método no se observaron pérdidas de actividad como consecuencia de posibles escapes de Radón. La actividad gamma procedente de la radiación cósmica fue medida “in situ”, a la toma de muestras, mediante un sistema de recuento de centelleo sólido de $\mathrm{INa}(\mathrm{Tl})$ Las conversiones de las velocidades de recuento alfa, beta y cósmica a tasas de dosis, han sido realizadas en base a los estudios de Nambí y Aitken (1986). 
Los errores asociados a las edades estimadas tienen en cuenta tanto los errores sistemáticos como estadísticos correspondientes a las medidas de $\mathrm{Tl}$, velocidades de dosis establecidas y procesos de calibrado de las fuentes radioactivas y equipos utilizados. El cálculo de dichos errores ha sido realizado en base a los estudios de Aitken (1985) y Arribas et al. (1990).

\section{RESULTADOS}

Los resultados obtenidos, correspondientes a las distintas muestras estudiadas (Tabla I), muestran claramente que el último proceso de calentamiento enérgico de las muestras cerámicas, tuvo lugar entre $6486 \pm 577$ y $6089 \pm 529$ años B.P. Lo cual conduce a situar el momento de fabricación de las mismas en el quinto milenio A.C.

En las tablas II y III se muestran los datos técnicos realizados en el proceso de datación de estas muestras, y a partir de los cuales se llega a las fechas establecidas anteriormente, con sus correspondientes incertidumbres.

Tabla I.- Resultados de las medidas de TL.

\begin{tabular}{|c|c|c|c|c|}
\hline Muestra & $\begin{array}{c}\text { Dosis Arqueo. } \\
(\mathrm{ED}+\mathrm{I} \text { Gy) }\end{array}$ & $\begin{array}{c}\text { Dosis Anual } \\
(\mathrm{mGy} / \mathrm{a})\end{array}$ & $\begin{array}{c}\text { Fecha } \\
\text { (años B.P.) }\end{array}$ & $\begin{array}{c}\text { Localización } \\
\text { Abrigo del Espino }\end{array}$ \\
\hline MAD-1048 & 115,23 & 17,97 & $6409 \pm 563$ & $\begin{array}{c}\text { Cerámica Mamelón } \\
\text { (Inv. 18) }\end{array}$ \\
\hline MAD-1049 & 98,25 & 16,07 & $6486 \pm 577$ & $\begin{array}{c}\text { Cerámica Lisa } \\
\text { (Inv. 19) }\end{array}$ \\
\hline MAD-1050 & 161,63 & 27,67 & $6089 \pm 529$ & $\begin{array}{c}\text { Cerámica Incisa } \\
\text { (Inv. 20) }\end{array}$ \\
\hline MAD-1051 & 140,08 & 23,5 & $6128 \pm 598$ & $\begin{array}{c}\text { Cerámica con Colorante } \\
\text { (Inv. 11) }\end{array}$ \\
\hline MAD-1052 & 159,89 & 2,09 & $6323 \pm 738$ & $\begin{array}{c}\text { Cerámica Impresa } \\
\text { (Inv. 6) }\end{array}$ \\
\hline
\end{tabular}

\section{DATOS TÉCNICOS}

Tabla II.- Medidas de TL

\begin{tabular}{|c|c|c|c|c|c|}
\hline Muestra & $\begin{array}{c}\text { Dosis Equi.+ Desvi. Estan. } \\
\text { Ed(Gy) }\end{array}$ & $\begin{array}{l}\text { Supralinealidad } \\
\text { I (Gy) }\end{array}$ & Plateau & $\begin{array}{c}\text { Valor } \\
\mathrm{K}\end{array}$ & Condiciones \\
\hline MAD-1048 & $115,23 \pm 8,5$ & 0 & $300-350^{\circ} \mathrm{C}$ & 1,71 & \multirow{5}{*}{$\begin{array}{c}\text { Tamaño Grano } \\
2-10 \mu \\
\text { Precalentamiento } \\
90^{\circ} \mathrm{C} \\
\text { Durante } 120 \mathrm{sg} .\end{array}$} \\
\hline MAD-1049 & $98,25 \pm 16,12$ & 0 & $300-350^{\circ} \mathrm{C}$ & 0,82 & \\
\hline MAD-1050 & $161,63 \pm 23,55$ & 0 & $300-350^{\circ} \mathrm{C}$ & 1,76 & \\
\hline MAD-1051 & $140,08 \pm 19,62$ & 0 & $300-350^{\circ} \mathrm{C}$ & 1,62 & \\
\hline MAD-1052 & $159,89 \pm 20,14$ & 0 & $300-360^{\circ} \mathrm{C}$ & 2,00 & \\
\hline
\end{tabular}


Tabla III.- Medida de la Tasa de Dosis

\begin{tabular}{|c|c|c|c|c|c|c|c|c|c|}
\hline Muestra & $\begin{array}{c}\text { Tasa dosis } \\
(\mathrm{mGy} / \mathrm{a})\end{array}$ & $\alpha \%$ & $\mathrm{~B} \%$ & $\gamma \%$ & $\begin{array}{c}\text { Radón } \\
\%\end{array}$ & $\begin{array}{c}\text { Rad. Cos } \\
(\mathrm{mGy})\end{array}$ & $\begin{array}{c}\text { Agua } \\
\text { Muestra } \\
\%\end{array}$ & $\begin{array}{c}\text { Agua } \\
\text { Medio } \\
. \%\end{array}$ & $\begin{array}{c}\text { Agua } \\
\text { Sust. Muestra } \\
. \%\end{array}$ \\
\hline $\begin{array}{c}\text { MAD-1048 } \\
\text { Cer. Mamelon }\end{array}$ & 17,97 & 75 & 12 & 13 & 0 & 1,29 & 8,97 & 8,97 & 14 \\
\hline $\begin{array}{c}\text { MAD-1049 } \\
\text { Cer. Lisa }\end{array}$ & 16,07 & 68 & 16 & 15 & 0 & 1,29 & 1,97 & 1,97 & 13,18 \\
\hline $\begin{array}{c}\text { MAD-1050 } \\
\text { Cer. Incisa }\end{array}$ & 27,67 & 80 & 10 & 9 & 0 & 1,29 & 2,75 & 2,75 & 10,48 \\
\hline $\begin{array}{c}\text { MAD-1051 } \\
\text { Cer. Colorante }\end{array}$ & 25,5 & 81 & 9 & 10 & 0 & 1,29 & 0,86 & 0,86 & 16,42 \\
\hline $\begin{array}{c}\text { MAD-1052 } \\
\text { Cer. Impresa }\end{array}$ & 25,62 & 80 & 10 & 10 & 0 & 1,29 & 0,3 & 0,3 & 25,47 \\
\hline
\end{tabular}

\section{COMENTARIO}

Se ha hecho referencia a las analogías entre las cerámicas del abrigo de El Espino y otros conjuntos segovianos, tanto en el propio barranco del Duratón como en las márgenes del Pirón, donde se emplaza la cueva de La Vaquera (Torreiglesias), uno de los yacimiento más señeros y mejor conocidos del Neolítico de la Meseta. Las técnicas decorativas y la profusión y sintaxis ornamental de nuestra exigua muestra, fruto del azar, coinciden con las caracterización e individualidad de la Fase I del Neolítico de La Vaquera que, según $\mathrm{M}^{a}$. S. Estremera (1999:247) "representa el primer horizonte del neolítico del yacimiento y también su primera ocupación". El marco temporal, en cronología absoluta, hay que situarlo entre $6120 \pm 160$ (nivel 104 adscrito a la Fase I) y 5800 \pm 30 b.p. (nivel 90) correspondiente ya a la Fase II (Estremera 1999:249).

Las analogías formales, técnicas y cronológicas nos llevan a concluir que la datación por TL de las cerámicas recogidas al aire libre, de contexto incierto, al resguardo del abrigo de El Espino, son coherentes con las aportadas por La Vaquera y con las novedades relacionadas con los contextos más antiguos del Neolítico en tierras de la Meseta (Estremera 1999; Kunst y Rojo 1996 y 1999; Jiménez Guijarro 1998 y 1999; Rojo y Kunst 1999) siempre que la equiparidad se haga entre las dataciones radiocarbónicas no calibradas y nuestros resultados por $T L$, lo que nos lleva a postular que el asentamiento de los grupos productores que "colonizan" la Meseta, es decir, el comienzo del Neolítico en estas tierras del interior peninsular, tuvo lugar en un tiempo relativamente corto cuyo apogeo se centra en la segunda mitad del $\mathrm{V}^{\circ}$ milenio a $\mathrm{C}$.

Una conclusión final de estas páginas es la llamada de atención sobre la fiabilidad de la TL como método de datación, frente a la problemática de las calibraciones por datación C14. Pese a la aparente desviación y márgenes de las cinco muestras analizadas, la realidad, a la escala en que nos movemos, es la homogeneidad del resultado en una banda temporal bastante corta-entre $6486 \pm 577$ y $6089 \pm 529$ años B.P. - máxime si tenemos en cuenta que los fragmentos estaban todos a la intemperie, erosionados y expuestos a las inclemencias atmosféricas.

De aquí se desprende otra conclusión, la potencialidad que brinda la termoluminiscencia para obtener dataciones muy concretas, a partir de un documento arqueológico tan omnipresente en el registro arqueológico 
como es la cerámica o la arcilla sometida a cocción, con las ventaja añadida, frente a otros métodos o muestras, de que la cronología asignada corresponde, bien al proceso de fabricación, al momento en que el producto humano sufrió la acción físico/química del fuego, bien a algún episodio, que puede ser muy próximo, relacionado con un calentamiento enérgico superior incluso al grado de cocción.

Las dataciones absolutas que por este método de TL, y en el mismo Laboratorio, se han obtenido de otras tantas cerámicas madrileñas procedentes de la Colección Bento, y cuyas primicias nos da a conocer la Dra. Rubio en este mismo Homenaje, abundan, en lo dicho:

a) La correspondencia entre fechas de TL y radiocarbónicas, para un material cerámico susceptible de encuadrarse, por simple analogía, en los primeros momentos del Neolítico de la Meseta.

b) La posibilidad de recurrir al método para ordenar temporalmente testimonios materiales de ignorado o problemático contexto.

\section{BIBLIOGRAFÍA CITADA EN EL INFORME DEL LABORATORIO DE DATACIÓN Y RADIOQUÍMICA DE LA UAM}

AITKEN, M.J. (1985): TL Dating. Academy Press, London

ARRIBAS, J.G.; MILLÁN, A.; SIBILA, E. y CALDERÓN, T. (1990): "Factores que afectan en la determinación del error asociado a la datación absoluta por TL: Fábrica de Ladrillos", Bol. Soc. Es. De Min. 13: 141-147.

FLEMINGS, S. L. (1970): “Thermoluminescen Dating. Refinement of Quartz Inclusion Method”, Archeometry 12: $13-30$.

NAMBI, K.S.V. y AITKEN, M.J. (1986): "Annual dose conversion factors for TL and ESR Datind", Archaeometry 28: 202-205.

ZIMMERNMAN, D.W. (1971): "Thermoluminiscent dating using fine grain from pottery", Archaeometry 13: 29-52.

URBINA, M.; MILLÁN, A., BENEITEZ, P. y CALDERÓN, T.(1997): “Minerales potencialmente útiles para el cálculo de dosimetrías retrospectivas en alimentos “, Alimentaria, 97, 53-59

\section{BIBLIOGRAFÍA CITADA EN EL TEXTO, RELATIVA AL NEOLÍTICO DE LA MESETA}

ESTREMERA, M ${ }^{a}$ S. (1999): "Sobre la trayectoria del Neolítico Interior: Precisiones a la secuencia de la Cueva de La Vaquera (Torreiglesias, Segovia), Il Congrés del Neolitíc a la Península Ibérica, SAGUNTUM-PLAV, Extra-2: 245-250.

JIMÉNEZ GUIJARRO, J. (1998): “ La neolitización de la cuenca alta del Tajo: nuevas propuestas interpretativas para el Neolítico de la Meseta", Complutum 9: 21-42

JIMÉNEZ GUIJARRO, J. (1999):El proceso de neolitización del interior peninsular II Congrés del Neolitíc a la Península Ibérica, SAGUNTUM-PLAV, Extra-2: 493-501.

KUNST, M. y ROJO, M. (1999): "El Valle de Ambrona: Un ejemplo de la primera colonización Neolítica de las tierras del Interior Peninsular", II Congrés del Neolitíc a la Península lbérica, SAGUNTUM-PLAV, Extra-2: 259-270.

LUCAS, M ${ }^{a}$ R.; ANCIONES, R.; CARDITO, L.M.; ETZEL, E. y RAMÍREZ, I. (1997): “Neolítico y arte rupestre en el Barranco del Duratón, Segovia” en R. Balbín y P. Bueno (eds.) II ${ }^{\circ}$ Cong. Arq. Peninsular, t. II: 157-163. Zamora. 
LUCAS, Mª R.; CARDITO, L. M.; ETZEL, E.; ANCIONES, R. y RAMÍREZ, I. (2001): “El Solapo del Águila: Monumento salvaje en la geografía simbólica en el Barranco del Duratón (Segovia)", Actas Congreso Internacional de Arte Rupestre Europeo, Concello de Vigo (versión en C.D.)

MUNICIO, L. y RUIZ GÁLVEZ, M. (1986): "Un nuevo yacimiento Neolítico en la Meseta Norte: las cerámicas decoradas de la cueva de la Nogalera (Villaseca, Segovia)", Numantia II: 143-148.

ROJO, M.A. y KUNST, M. (1999): "La Lámpara y la Peña de la Abuela. Propuesta secuencial del Neolítico Interior en el ámbito funerario", II Congrés del Neolitíc a la Península Ibérica, SAGUNTUM-PLAV, Extra-2: 503-512.

ROJO, M.A. y KUNST, M. (1996): "Proyecto de colaboración hispano-alemán en torno a la introducción de la neolitización en las tierras del interior peninsular: planteamientos y primeros resultados", CuPAUAM 23: 87-113. 

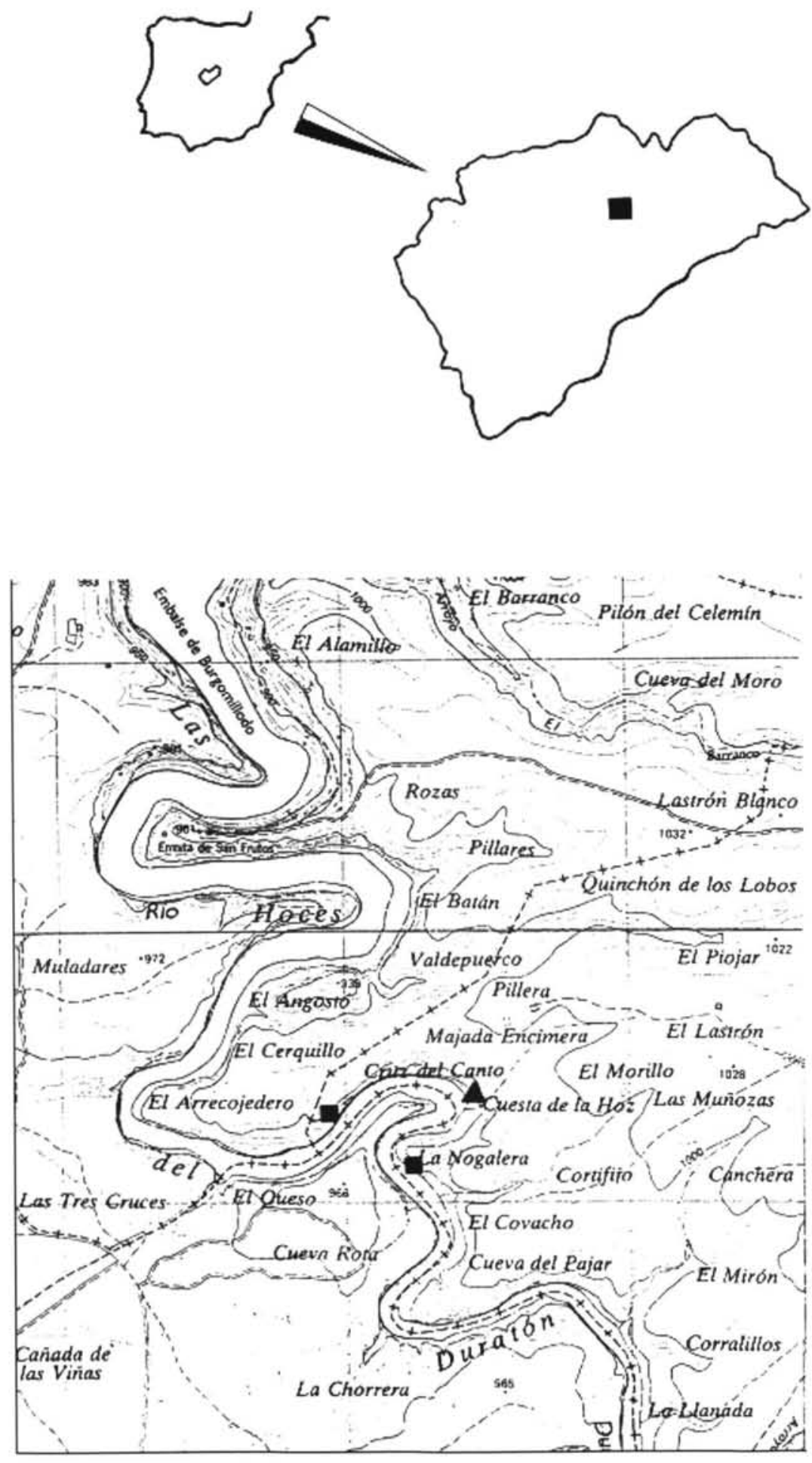

Fig. 1: $\boldsymbol{\Delta}$ Localización del abrigo del Espino (Villaseca, Segovia)

Localización de la Cueva de la Nogalera (Neolítico y Arte Esquemático) y del Solapo del Águila (Arte Esquemático) 

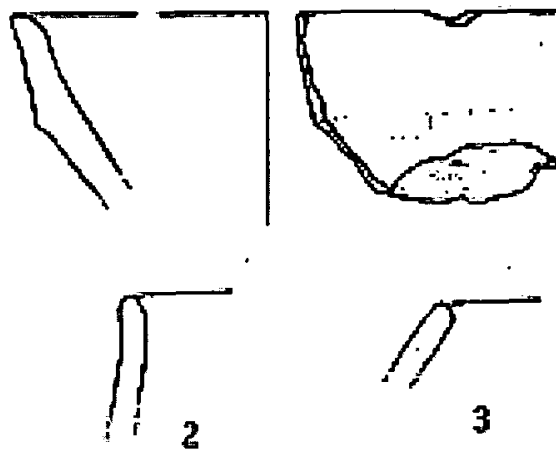

3

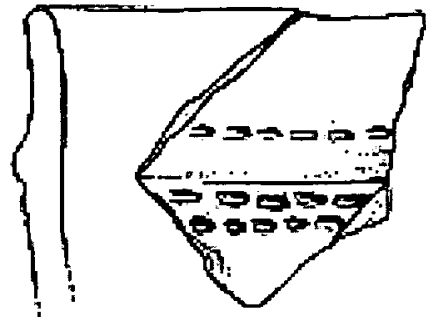

5
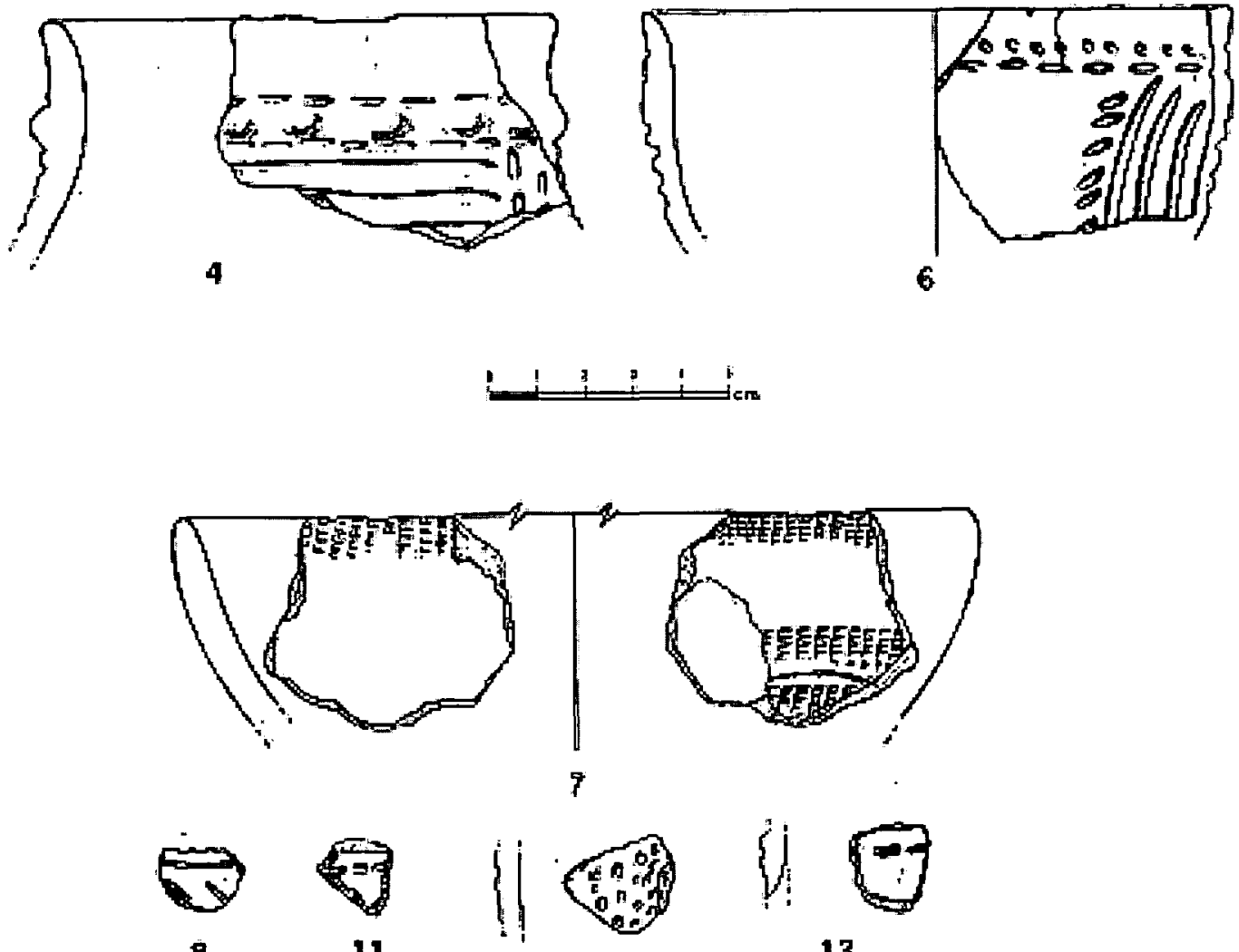

$\begin{cases}1 & -7 \\ 11 & -7\end{cases}$

8

11

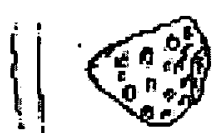

12

13
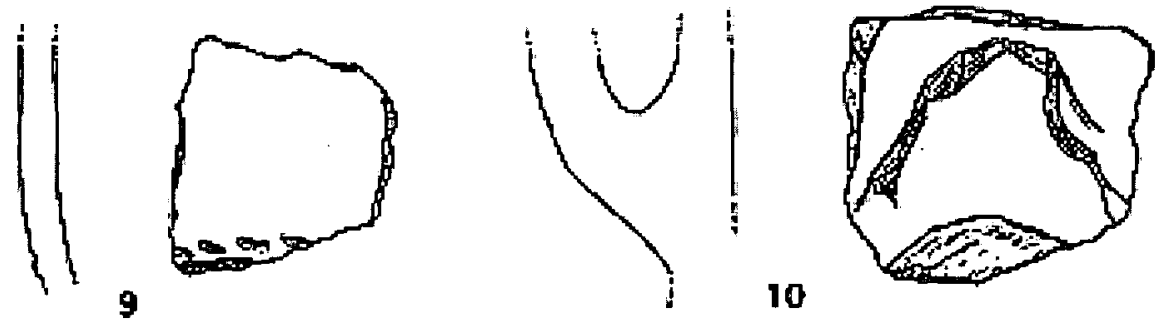

Fig. 2: Fragmentos cerámicos recogidos en el abrigo dèl Espino (núm. 6: incrustación blanca; 8, 11 y 12: incrustación roja)

Núm.6: TL Mad-1052; núm.11: TL Mad-1051. 


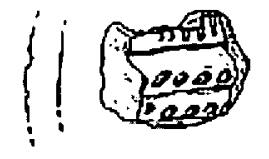

14

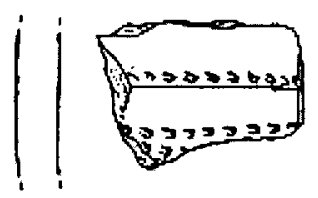

15

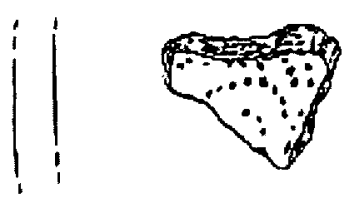

16

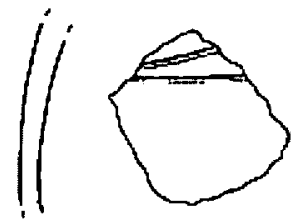

17

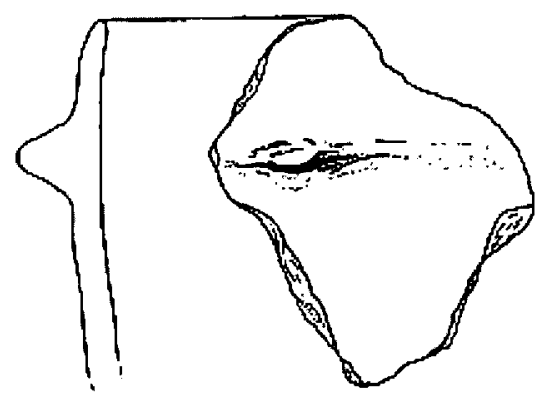

18

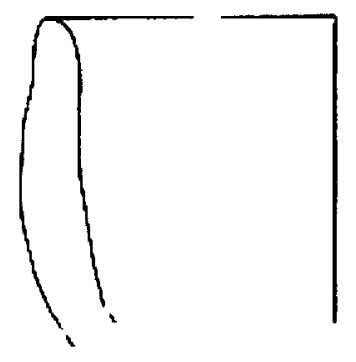

19

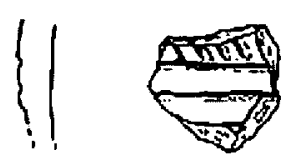

20

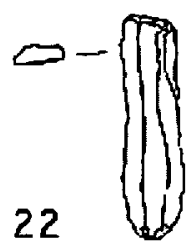

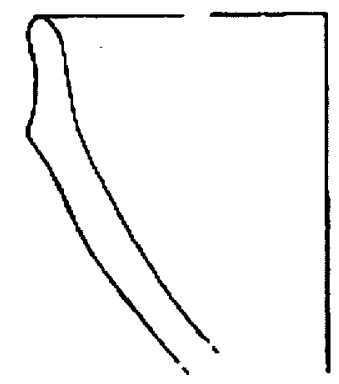

21

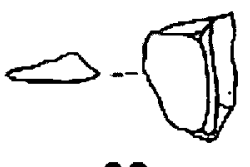

23

Fig. 3: Fragmentos cerámicos recogidos en el abrigo del Espino (núm 15: incrustación roja; núm.18 y 20: ¿superficies a la almagra?). Núm. 22 y 23: material lítico.

Núm.18: TL Mad-1048; núm.19: TL Mad-1049; núm.20: TL Mad-1050. 